\title{
Simultaneous Adsorption of Cation and Anion by Thermosensitive Hydrogels
}

\author{
Toni SuHARTO ${ }^{1}$, Takehiko GOTO ${ }^{*}$, and Satoshi NAKAI ${ }^{1}$ \\ ${ }^{1}$ Graduate School of Engineering, Hiroshima University, 1-4-1 Kagamiyama, Higashi-Hiroshima City, Hiroshima 739-8527, Japan
}

\begin{abstract}
Simultaneous usage of cationic and anionic thermosensitive hydrogels was shown to positively contribute to adsorption of heavy metal ions. The potential capacity for recovering heavy metal ions from an aqueous solution was enhanced by incorporating relatively hydrophobic moiety to the hydrogels, which was ascribed to the increase in the desorption on elevating the temperature over the lower critical swelling temperature (LCST). N-tert-butylacrilamide was added into the hydrogels to increase its desorption potential. The addition of N-tert-butylacrylamide does not significantly affect hydrogels' adsorption ability. Moreover, the adsorption-desorption cycle was not diminished by repeating the temperature swing above the critical temperature at which the volume phase transition was induced.
\end{abstract}

\section{Introduction}

Rapid industrial development is one of the causes of increasing metal concentrations in the environment. Since heavy metals are non-biodegradable and persistent, this problem need more attention. The presence of heavy metals, especially in the form of liquid waste, is a problem that needs serious attention. In certain concentration, it can results in toxic and dangerous effects, both in the aquatic ecosystem and the surrounding human life.

Between 2000 and 2009, a massive expansion of the coal industry occurred in Indonesia. Indonesia developed the world's fastest growing coal sector (Greenpeace South East Asia-Indonesia, 2014). A study of acid mine drainage (AMD) in South Kalimantan found considerable sources of AMD and several examples of low $\mathrm{pH}$ in the nearby ponds. The study revealed that in Binuang area, South Kalimantan Province, coal mining produced waste dumps and AMD in many settlement ponds with a considerably low $\mathrm{pH}$ (2.8 to 4.4$)$, with metals among others magnesium, manganese, iron, cadmium, hexavalent chromium and lead (Simarmata et al., 2011). These heavy metals pose a serious threat to human health. The study found that mining wastewater had mixed with groundwater, surface water, and rainwater. Most of the issues come from the untreated used mining sites. As the rain comes, those cavities will hold water that will mix with all heavy metals that are left in the sites (Greenpeace South East AsiaIndonesia, 2010). For example, lead poisoning can lead to a various range of health impact range from sub-clinical effects (e.g., subtle hematologic changes) to lethal pathologic alteration (e.g., nervous system failure and death) (Plaza and Lambetucci, 2019). Since mining activities at this site are ongoing, the study concluded that the mine would continue to produce AMD in years to come, life-threatening problems that need to be taken seriously.

To date, many technologies have been developed to reduce the level of heavy metals contaminant in industrial wastewater. The conventional method commonly used in the separation of industrial wastewater heavy metals is precipitation and neutralization (Rubio et al., 2002). But this method produces secondary waste in the form of sludge with high concentrations of heavy metal ions. In addition to the above methods, alternative methods have been developed including Reverse Osmosis (RO), Nano Filtration (NF) which uses membranes as a medium for the separation of heavy metals with liquid waste but the disadvantages of this method require high operational costs. The use of adsorbents containing ligands such as ion-exchange groups or chelating agents has a weakness during the regeneration process of cation and anion resins because they require strong acids and strong bases. This process if not handled properly will cause environmental pollution due to secondary waste in the form of strong acids/bases (Qdais and Moussa, 2004). Adsorption by using thermosensitive ionic hydrogels provides a promising solution to overcome those problems since it doesn't produce any byproducts other than recycled gels.

Several researchers suggested that adsorption of heavy metal by ionic hydrogel might be possible by utilizing the right monomers. Adsorption of heavy metal by thermosensitive ionic hydrogels, such as zwitterionic betaine, can recover few amounts of heavy metal ions, due to the interactions between cationic and anionic group located in the same unit loop in sulfobetaine polymer (Neagu et al., 2010). But the adsorption of that type of gels is hindered by the inter-chain, intra-chain, and intra-

Corresponding author: tgoto@hiroshima-u.ac.jp 
group linkage that happen due to having 2 different charged group in one chain. Those linkage limits gels' ability to swell thus, decreasing its adsorption ability (Ningrum et al., 2014).

To solve those issues in this research, cationic and anionic gels of adsorbent and $N$-isopropyl acrylamide (NIPAM) were synthesized and utilized separately. PolyNIPAM is one example of a thermosensitive polymer with a temperature of Low Critical Solution Temperature (LCST) around $32^{\circ} \mathrm{C}$ (Hirokawa and Toyoichi, 1984). NIPAM undergoes swelling at low temperatures and shrinking at high temperatures because of its transition from hydrophilic to hydrophobic and has a neutral charge (Ju et al., 2009). With NIPAM as the major constituent of the gels, it is possible to adsorb and desorb heavy metal by temperature swing.

\section{Methodology}

\subsection{Materials}

$N$-isopropylacrylamide (NIPAM) and $N, N$-dimethyl aminopropylacrylamide (DMAPAA) were kindly supplied by KJ Chemical Co. Japan. 2-acrylamido-2methylpropanesulfonic acid (AMPS) was obtained from Tokyo Chemical Industry Co., Ltd. Acrylic acid (AAc) was obtained from Katayama Chemical Japan. Tetramethylethylenediamine (TEMED) was obtained from Nacalai Tesque Inc. Japan. $N$-tert-butylacrylamide (NTBA), $N, N$ '-methylenebis(acrylamide) (MBAA) and ammonium persulfate (APS) was obtained from Sigma Aldrich Co. USA. 1,3-propanesultone (PS) was obtained from Kanto Chemical Inc. Japan. NIPAM and DMAPAA were purified before use. The other reagents were of analytical grade and can be used without further purification.

\subsection{Synthesis of DMAAPS}

DMAAPS was synthesized by following ring opening reaction of DMAPAA and PS. A mixture of PS (75g) and Acetonitrile $(75 \mathrm{~g})$ was added dropwise to a mixture of 100g DMAPAA and $200 \mathrm{~g}$ acetonitrile while being stirred at $30^{\circ} \mathrm{C}$ for $90 \mathrm{~min}$. The mixture was continuously stirred for $16 \mathrm{~h}$, then the mixture was allowed to precipitate for $48 \mathrm{~h}$. The precipitated crystals were filtered and washed with acetone to remove unreacted reagents. Finally, DMAAPS were dried using an oven at $50^{\circ} \mathrm{C}$ (Ningrum et al., 2014).

\subsection{Polymerization of ionic hydrogels}

All hydrogels were synthesized by free radical polymerization method. Monomers, cross-linker, and accelerator were mixed in one container, while initiator was prepared in a separate container. Total solution's volume was $25 \mathrm{~mL}, 5 \mathrm{~mL}$ of APS as initiator solution and $20 \mathrm{~mL}$ for a mixture of NIPAM, DMAPAA, AMPS, AAc, DMAAPS, NTBA as monomers, MBAA as cross-linker, and TEMED as accelerator. Due to the hydrophobicity of
NTBA, 75\% ethanol solution was used to dissolve all reactants. Reaction was conducted at $10^{\circ} \mathrm{C}$ for 24 hours after 1 hour of purging. After formed, gels were washed with methanol using soxhlet extractor for 24 hours.

Table 1. Composition of Ionic Hydrogels

\begin{tabular}{ccccc}
\hline \multirow{3}{*}{ Monomer } & \multicolumn{5}{c}{ Concentration $(\mathrm{mol} / \mathbf{L})$} \\
\cline { 2 - 5 } & $\begin{array}{c}\text { NIPAM } \\
\text { DMAPAA } \\
\text { (ND) }\end{array}$ & $\begin{array}{c}\text { NIPAM } \\
\text { AMPS } \\
\text { (NS) }\end{array}$ & $\begin{array}{c}\text { NIPAM } \\
\text { AAC } \\
\text { (NA) }\end{array}$ & $\begin{array}{c}\text { NIPAM } \\
\text { DMAAPS } \\
\text { (NAs) }\end{array}$ \\
\hline N & 0.9 & 0.9 & 0.9 & 0.9 \\
D & 0.1 & 0 & 0 & 0 \\
S & 0 & 0.1 & 0 & 0 \\
A & 0 & 0 & 0.1 & 0 \\
As & 0 & 0 & 0 & 0.1 \\
NT & 0 & 0 & 0 & 0 \\
MB & 0.05 & 0.05 & 0.05 & 0.05 \\
\hline $\mathrm{N}=$ NIPAM, D = DMAPAA, S = AMPS, A = AAc,
\end{tabular}

As $=$ DMAAPS, NT $=$ NTBA, $M B=$ MBAA

Table 2. Composition of NTBA Cationic Hydrogels

\begin{tabular}{|c|c|c|c|c|c|c|c|c|}
\hline \multirow{3}{*}{$\begin{array}{c}\text { Mo } \\
\text { no } \\
\text { mer }\end{array}$} & \multicolumn{8}{|c|}{ Concentration $(\mathrm{mol} / \mathrm{L})$} \\
\hline & \multicolumn{2}{|c|}{ NTBA0 } & \multicolumn{2}{|c|}{ NTBA100 } & \multicolumn{2}{|c|}{ NTBA200 } & \multicolumn{2}{|c|}{ NTBA300 } \\
\hline & Cat & Ani & Cat & Ani & Cat & Ani & Cat & Ani \\
\hline $\mathrm{N}$ & 0.9 & 0.9 & 0.8 & 0.8 & 0.7 & 0.7 & 0.6 & 0.6 \\
\hline $\mathrm{D}$ & 0.1 & 0 & 0.1 & 0 & 0.1 & 0 & 0.1 & 0 \\
\hline $\mathrm{S}$ & 0 & 0.1 & 0 & 0.1 & 0 & 0.1 & 0 & 0.1 \\
\hline NT & 0 & 0 & 0.1 & 0.1 & 0.2 & 0.2 & 0.3 & 0.3 \\
\hline $\mathrm{MB}$ & 0.05 & 0.05 & 0.05 & 0.05 & 0.05 & 0.05 & 0.05 & 0.05 \\
\hline
\end{tabular}

Finally, the gels were air-dried for 1 day then followed by oven drying at $50^{\circ} \mathrm{C}$ for 1 day. The concentration of each monomer on each type of gels are given in Table 1 and Table 2. Dried gels were ground until it reaches a particle diameter of $0.5-1 \mathrm{~mm}$.

\subsection{Hydrogels' water intake}

In order to compare each gels' performance, 6 samples were prepared NIPAM-DMAPAA (ND), NIPAM-AMPS (NS), NIPAM-AAc (NA), NIPAM-DMAAPS (NAs), NIPAM-DMAPAA + NIPAM-AMPS (ND+NS), and NIPAM-DMAPAA + NIPAM-AAc (ND+NA). Amount of water absorbed by the hydrogels was measured by comparing the initial amount of solution with the solution's volume at the equilibrium condition. For $15 \mathrm{~mL}$ of $5 \mathrm{mM} \mathrm{PbCl} 2$ solution, a total of $0.12 \mathrm{~g}$ of hydrogels were added into the solution. For ND+NS and ND+NA, the ratio of cationic gels to anionic gels was 1 to 1 . In Figure 1 the experimental scheme of simultaneous usage of cationic and anionic hydrogels can be seen. The samples were continuously shaken at $100 \mathrm{rpm}$ at the set temperature while the sorption tests were carried out for $24 \mathrm{~h} .25^{\circ} \mathrm{C}$ was chosen for the adsorption and $50^{\circ} \mathrm{C}$ for desorption. Solution and gels were separated using a paper filter, then filtrate's volume was measured using the following formula.

$$
Q_{w}=\frac{V_{0}-V_{e}}{m}
$$

Where $\mathrm{Q}_{\mathrm{w}}$ is the amount of water adsorbed by hydrogels $\left(\mathrm{mL} / \mathrm{g}\right.$ dry gels). $\mathrm{V}_{0}$ is the initial volume of the solution 
$(\mathrm{mL}) . \mathrm{V}_{\mathrm{e}}$ is solution's volume at equilibrium condition (mL). $m$ is the mass of dry gels $(\mathrm{g})$.

\subsection{Adsorption and desorption of ions}

Amount of heavy metal ion adsorbed by the hydrogels was measured by comparing the initial concentration the equilibrium concentration. The same experimental conditions as the water intake experiment were used for this experiment.

$$
Q=\frac{C_{0} V_{0}-C_{e} V_{e}}{m}
$$

Where $Q$ is the amount of heavy metal adsorbed by hydrogels ( $\mathrm{mmol} / \mathrm{g}$ dry gel). $V_{0}$ is the initial volume of the solution $(\mathrm{mL}) . V_{e}$ is solution's volume at equilibrium condition $(\mathrm{mL}) . C_{0}$ and $C_{e}$ are initial and equilibrium concentration of heavy metal ions respectively $(\mathrm{mmol} / \mathrm{L})$. $m$ is the mass of dry gels $(\mathrm{g})$.

For desorption tests, gels were allowed to adsorb ions in $5 \mathrm{mM} \mathrm{PbCl}_{2}$ solution at $25^{\circ} \mathrm{C}$ for 24 hours. Then the temperature was changed to $50^{\circ} \mathrm{C}$ to desorb recovered ions for 24 hours. As for NTBA gels there were 4 samples prepared, all of the samples were a mixture of NTBA cationic gel and anionic gel with 1:1 mass ratio.

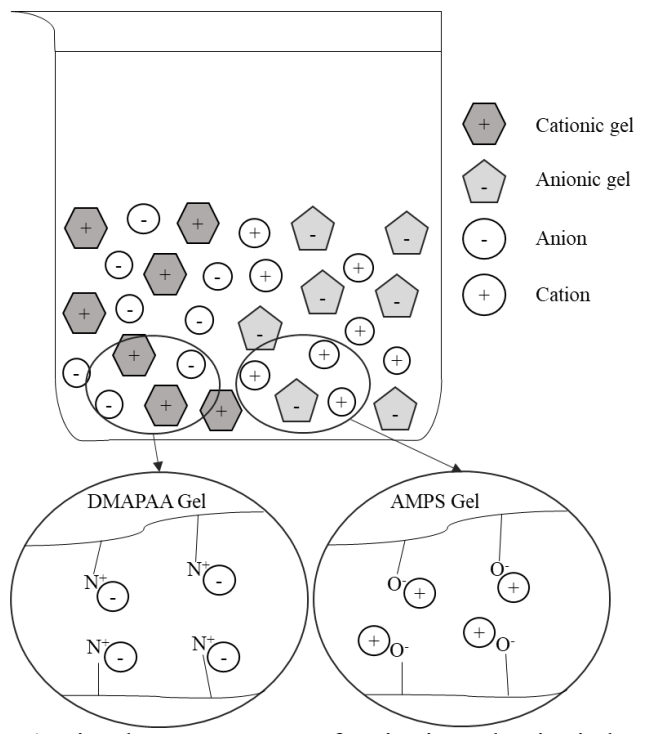

Figure 1. Simultaneous usage of cationic and anionic hydrogels

\subsection{Reversibility test}

By using the same conditions for adsorption and desorption tests, each gel was allowed to adsorb ions at $25^{\circ} \mathrm{C}$ and desorb recovered ions at $50^{\circ} \mathrm{C}$ for 24 hours each. This process was repeated 4 times. In this experiment, gels and solution, was kept in its equilibrium condition for each temperature, without any changes the mother solution.

\subsection{Inductively coupled plasma atomic emission spectroscopy test}

Heavy metal concentration in every sample was determined by Inductively Coupled Plasma Atomic Emission Spectroscopy (ICP-AES) using ICP SPS 3510
(Seiko Instruments Inc.). Every sample was diluted 20 times. Argon gas was used to measure the concentration of the solutions.

\subsection{Titration Test}

This test was conducted to observe gels' behavior in water/gel system. For $50 \mathrm{~mL}$ of $\mathrm{H}_{2} \mathrm{O} 0.1 \mathrm{~g}$ of the cationic gels was added to the solution. Then the slurry mixtures were left for 24 hours under controlled temperature and stirred at $100 \mathrm{rpm}$. $0.1 \mathrm{M} \mathrm{NaOH}$ was used as the titrant. Automatic titrator (COM-1700, Hiranuma, Japan) was used in this experiment.

\section{Result and Discussion}

\subsection{Water intake}

Figure 2 shows the amount of water can be absorbed by 6 different types of gels. As shown in Figure 2, simultaneous usage of cationic and anionic gels (ND+NA and ND+NS) provides higher water intake ability. Especially by using DMAPAA gel and AMPS gel $(\mathrm{ND}+\mathrm{NS})$

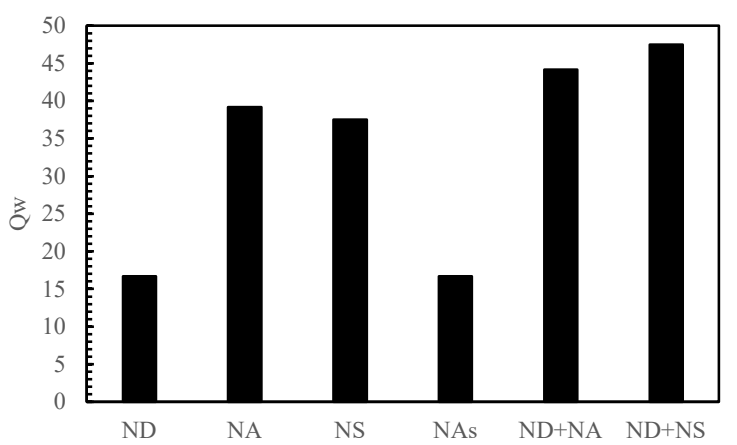

Figure 2. Water intake of various gels at $25^{\circ} \mathrm{C}$ in $5 \mathrm{mM} \mathrm{PbCl}_{2}$

Presence of sulfonate group gives AMPS a high degree of hydrophilicity at a wide range of $\mathrm{pH}$ (El-Hag et al., 2007). AMPS based hydrogels exhibit $\mathrm{pH}$-independent swelling behavior due to the presence of strongly ionizable sulfonic groups (Pourjavadi et al., 2007). By utilizing cation and anion gels separately, it allows the gel to absorb more water compared to another type of gels. Unlike the other simultaneous adsorption, NAs gels shows lower water intake compared to the ND+NS and ND+NA gels. This might be caused by an inter-chain, intra-chain, intra-group linkages that might happen within the gels' structure thus, inhibiting its ability to swell (Ningrum et al., 2015).

\subsection{Adsorption of ions}

Figure 3 shows the amount of cation and anion adsorbed by the gels. It needs to be noted that even cationic gel (ND) can adsorb cation through mass transfer promoted 
by the concentration gradient and anionic gels (NA and NS) are also able to adsorb anion as indicated in the Figure. NAs gel is a zwitterionic betaine gel, thus it contains both cation group and anion group in one chain. By having a positive charge and negative charge in one polymer molecule, entanglement between those charged groups may occur.

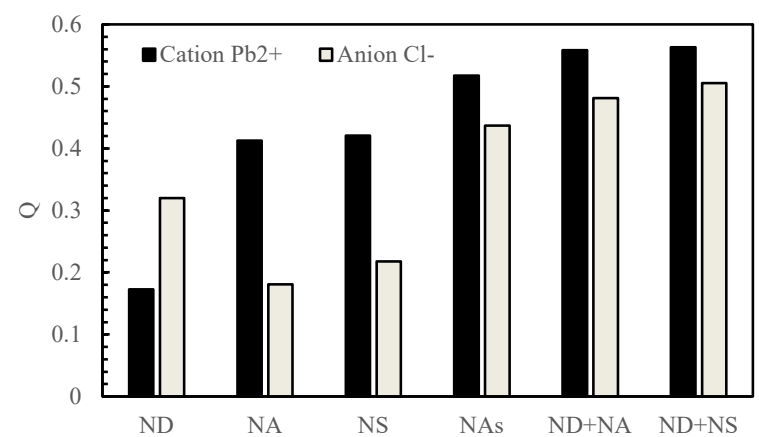

Figure 3. Adsorption of cation and anion at $25^{\circ} \mathrm{C}$ in $5 \mathrm{mM} \mathrm{PbCl}_{2}$

Due to the mentioned property, ions adsorption of NAs increased greatly but it is also hindered by the linkage mentioned before, which results in its inability to swell as shown in Figure 2. Based on the results in Figure 3, it can be concluded that by utilizing cationic and anionic gels to adsorb ions simultaneously, a higher recovery amount of ions and water intake ability can be obtained. Simultaneous adsorption using both cationic and anionic gels reduces the counter-ion effect. For example when only cations adsorbed, the opposing ions will inhibit the adsorption phenomena by competing with the adsorbent in bonding with the adsorbed ion (Zhu et al., 2018). However, the inhibition will occur especially when the amount of adsorbed cation and anion is not stoichiometrically balanced. Despite the inhibition, the overall adsorption ability is enhanced by the simultaneous usage of cationic and anionic gels.

\subsection{Desorption of ions}

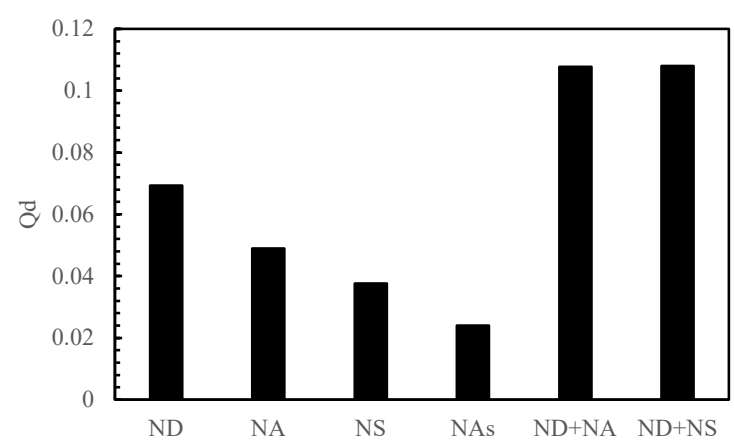

Figure 4. Desorption amount of $\mathrm{Pb}^{2+}$ by various gels at $50^{\circ} \mathrm{C}$ in $5 \mathrm{mM} \mathrm{PbCl}_{2}$

Since NIPAM is the major constituent of the gels, these gels are able to desorb recovered ions by temperature swing. Based on Figure 4, the combination of cationic and anionic gels yields the best result in desorbing recovered $\mathrm{Pb}^{2+}$ ions. While simultaneous adsorption enhances desorption ability, in the case of DMAAPS it is hindered. Due to the fact that the volume phase transition of NIPAM-DMAAPS as shown in Figure 2 is inhibited by the presence of intra-chain, inter-chain, intra-group entanglement.

\subsection{Addition of NTBA monomer}

Results from previous sections suggested that the combination of NIPAM-DMAPAA and NIPAM-AMPS gels gives the best result both for adsorption and desorption ability. Consequently, NTBA was introduced to NIPAM-DMAPAA and NIPAM-AMPS gels. As mentioned before, synthesized gels were able to desorb ions just by swinging temperature, but the desorbed amount was too small. To enhance gels desorption ability, hydrophobic NTBA monomers were introduced to the gels. By introducing NTBA to the existing gels, polymer's LCST will decrease as the concentration of NTBA gets higher (Save, 2003).

\subsubsection{Dissociation of ionic groups in the presence of water}

Figure 5 indicates that the addition of NTBA decreases $\mathrm{OH}^{-}$dissociation in the cationic gels as well as gels' LCST. NTBA gels stops to dissociate at a temperature above $30^{\circ} \mathrm{C}$. Based on the dissociation of $\mathrm{OH}$, the adsorption amount decreases with the increasing amount of NTBA concentration in the gels. Since heavy metal ions are attracted by the existing activated ionic groups within the gels, less amount of ionic group dissociated in the gels resulted in fewer ions adsorbed by gels. Moreover, by enlarging the differences in desorption temperature and LCST of gels, greater desorption ability may be obtained. Based on the result adsorption and desorption can be conducted at the same temperature as previous experiments

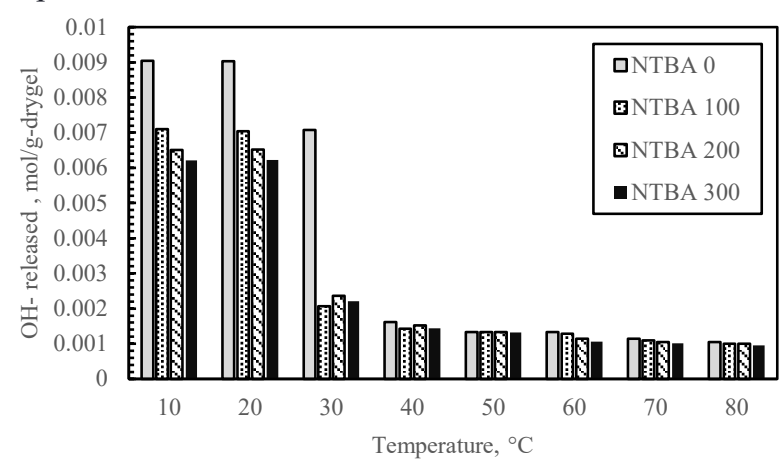

Figure 5. Dissociation of $\mathrm{OH}^{-}$in cationic NTBA gels

\subsubsection{Adsorption isotherm of NTBA gels}

Figure 6 a) and b) shows the adsorption isotherm of anionic NTBA gels at $25^{\circ} \mathrm{C}$ and $50^{\circ} \mathrm{C}$ respectively. As $\mathrm{Pb}^{2+}$ ion concentration increases, adsorption also increases due to large driving force in the concentration gradient. At $25^{\circ} \mathrm{C}$ gels adsorbs more than twice as much ions as at $50^{\circ} \mathrm{C}$, but at lower concentration, where driving force is relatively small, the adsorption amount is close to 
one another. This was caused by the activated adsorption sites that exist in the gels attracts opposing ions and adsorbs those ions. Moreover, adsorption sites increases as gels expands at lower temperature due to the dissociation of ionic groups as expressed in Figure 5. As gels adsorb water, gels' polymer network expands as well. The expansion results in bigger pores, allowing more ions to be transferred at higher solution concentration by an increase of driving force as mentioned before.
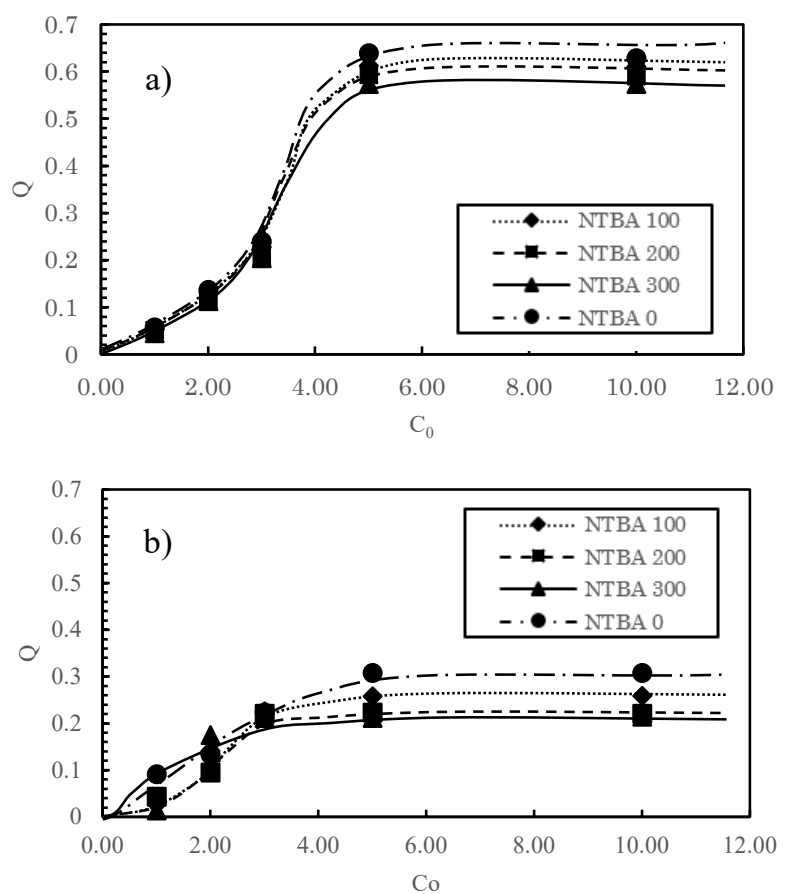

Figure 6. NTBA gels adsorption isotherm of $\mathrm{Pb}^{2+}$ at a) $25^{\circ} \mathrm{C}$ and b) $50^{\circ} \mathrm{C}$ in $5 \mathrm{mM} \mathrm{PbCl}_{2}$

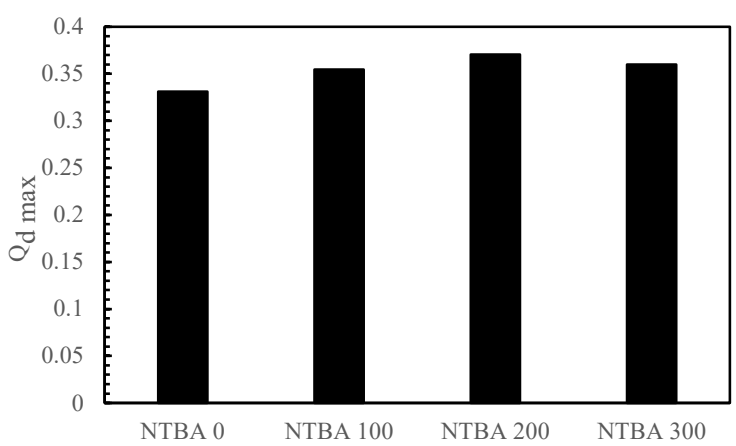

Figure 7. Anionic NTBA gels' maximum desorption potential of $\mathrm{Pb}^{2+}$ by temperature swing in $5 \mathrm{mM} \mathrm{PbCl}$.

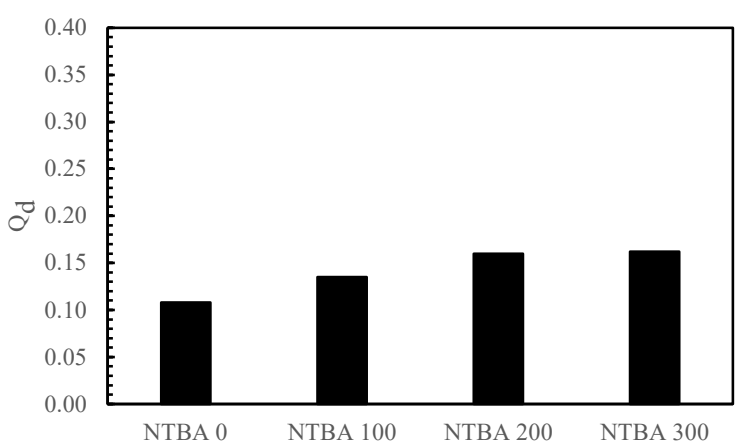

Figure 8. Anionic NTBA gels' actual desorption amount of $\mathrm{Pb}^{2+}$ by temperature swing in $5 \mathrm{mM} \mathrm{PbCl}_{2}$
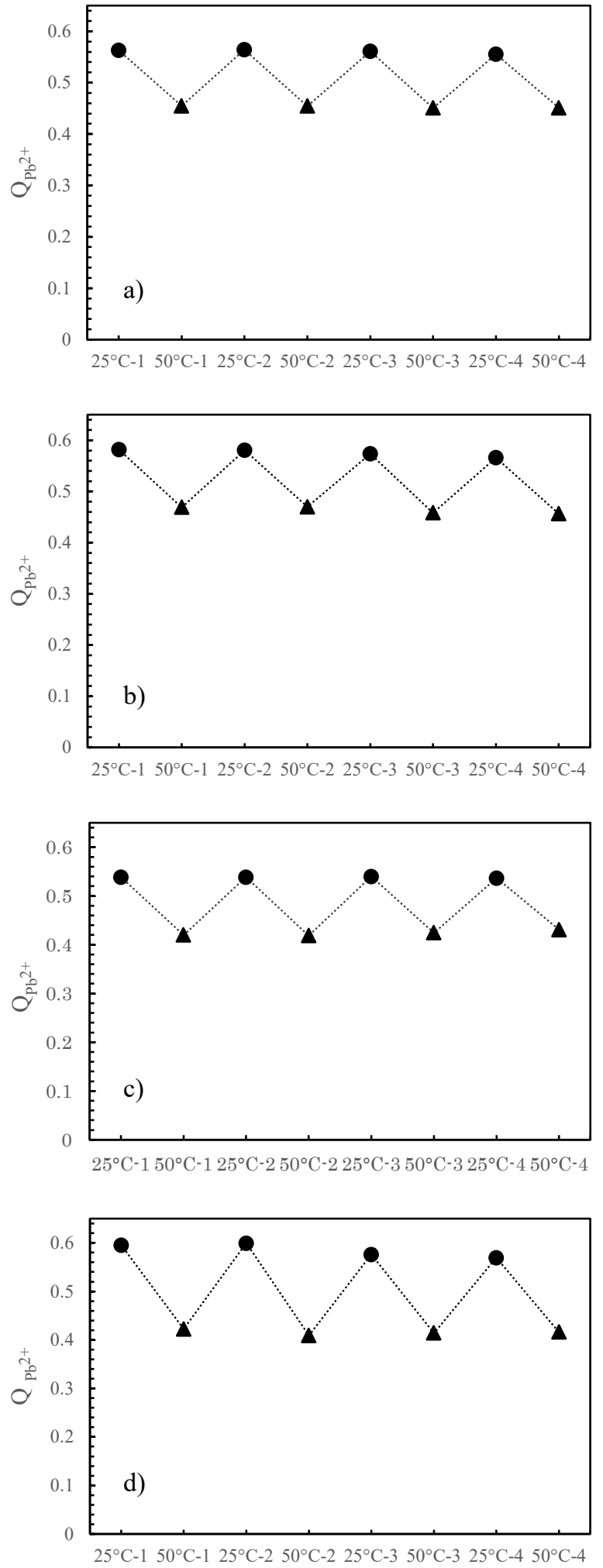

Figure 9. Reversibility test of a) NTBA 0 gel, b) NTBA 100 gel, c) NTBA 200 gel, and d) NTBA 300 gel in $5 \mathrm{mM}$ of $\mathrm{PbCl}_{2}$

\subsubsection{Desorption of NTBA gels}

Desorption potential of anionic NTBA gels is shown in Figure 7. By calculating the differences between the adsorption ability of $\mathrm{Pb}^{2+}$ at $25^{\circ} \mathrm{C}$ and $50^{\circ} \mathrm{C}$, maximum 
desorption ability of each hydrogel can be obtained through the following formula.

$$
Q_{\text {dmax }}=Q_{25^{\circ} \mathrm{C}}-Q_{50^{\circ} \mathrm{C}}
$$

Where $Q_{d \max }$ is the maximum desorption amount, $Q_{25^{\circ} \mathrm{C}}$ and $Q_{50^{\circ} \mathrm{C}}$ are adsorption ability of gels in $25^{\circ} \mathrm{C}$ and $50^{\circ} \mathrm{C}$ respectively. In Figure 8, the actual desorption amount of $\mathrm{Pb}^{2+}$ can be seen. The figure shows that the addition of NTBA increases gels' desorption ability. Due to the strong interaction between ions and the adsorbent, the actual desorption amount could only reach $30 \%$ of its desorption potential amount.

\subsubsection{Reversibility of NTBA gels}

Reversibility of each NTBA gel is shown in figure 9. Based on all of the results, it can be concluded that the addition of NTBA doesn't have any unfavorable effect on the gels' reusability. Nevertheless, since the addition of NTBA increases gels' hydrophobicity, the adsorption ability of NTBA gels slightly decreases while greatly enhancing the desorption ability of NTBA gels. With a higher hydrophobic NTBA concentration, the thermosensitive gels will desorb more ions. In the case of Figure 8, Changes to the desorption ability of NTBA gels can be seen with the changes in the value of $Q_{d}$. In this research, thermosensitive gels can be used up to 4 times without any significant loss in both its adsorption and desorption ability.

\section{Conclusion}

In the present work, cationic and anionic hydrogels consisted of thermo-sensitive NIPAM and adsorbent were successfully synthesized by free radical polymerization. Cationic and anionic gels exhibit the ability to adsorb heavy metal ions when they were used simultaneously. Above mentioned results indicates that by utilizing simultaneous adsorption, recovery amount of heavy metal can be enhanced. Moreover, with the addition of hydrophobic NTBA, desorption of ions by temperature swing increases as well. The thermosensitive gels can be recycled and utilized again without suffering significant efficiency loss. In general, these hydrogels have the potential to treat the heavy metal contaminated water in Kalimantan, Indonesia.

\section{Acknowledgements}

This research was supported by the JSPS KAKENHI Grant Number (17K06892).

\section{References}

El-Hag, A. A., H. A. A. El-Rehiem, E. S. A. Hegazy, and M. M. Ghoba-shy; "Characterization and Potential Application of Electro-Active Acrylamido-2-methyl Propane Sulfonic Acid/ Acrylic Acid Copolymer Prepared by Ionizing Radiation." Journal of Macromolecular Science, 44, 91-98 (2007)
Greenpeace South East Asia-Indonesia; Batubara Mematikan Bagaimana Rakyat Indonesia Membayar Mahal untuk Bahanbakar Terkotor di Dunia, p. 15, Greenpeace, Jakarta, Indonesia (2010)

Greenpeace South East Asia-Indonesia; Coal Mines Polluting South Kalimantan's Water, p. 24, Greenpeace, Jakarta, Indonesia (2014)

Hirokawa, Y. and T. Toyoichi; "Volume Phase Transition in a Nonionic Gel," Journal of Chemical Physics, 81, 7173 (1984)

Ju, X. J., S. B. Zhang, M. Y. Zhou, R. Xie, L. Yang, and L. Y. Chu; "Novel Heavy-Metal Adsorption Material: Ion-Recognition P(NIPAM-co-BCAm) Hydrogels for Removal of Lead(II) Ions," Journal of Hazardous Materials, 167, 114-118 (2009)

Neagu, V., S. Vasiliu, and S. Racovita; "Adsorption Studies of Some Inorganic and Organic Salts on New Zwitterionic Ion Exchangers with Carboxybetaine Moieties," Chemical Engineering Journal, 162, 965-973 (2010)

Ningrum, E. O., Y. Murakami, Y. Ohfuka, T. Gotoh, and S. Sakohara; "Investigation of Ion Adsorption Properties of Sulfobetaine Gel and Relationship with Its Swelling Behavior," Polymer, 55, 5189-5197 (2014)

Ningrum, E. O., Y. Ohfuka, T. Gotoh, and S. Sakohara; "Effects of Specific Anions on the Relationship between the Ion-Adsorption Properties of Sulfobetaine Gel and Its Swelling Behavior," Polymer, 59, 144-154 (2015)

Plaza P. I. and S. A. Lambetucci; "What Do We Know about Lead Contamination in Wild Vultures and Condors? A Review Decades of Research," Science of the Total Environment, 654, 409-417 (2019)

Pourjavadi, A., S. Barzegar, and F. Zeidabadi; "Synthesis and Properties of Biodegradable Hydrogels of $\kappa$ carrageenan Grafted Acrylic Acid-co-2-acrylamido-2methylpropanesulfonic Acid as Candidates for Drug Delivery Systems," Reactive and Functional Polymers, 67, 644-654 (2007)

Qdais, H. A. and H. Moussa; "Removal of Heavy Metal from Wastewater by Membrane Processes: A Comparative Study." Desalination, 164, 105-110 (2004)

Rubio, J., M. Souza and R. Smith; "Overview of Flotation as a Wastewater Treatment Technique," Minerals Engineering, 15, 139-155 (2002)

Save, N. S., M. Jassal, and A. K. Agrawal; "Stimuli Sensitive Copolymer Poly(N-tert-butylacrylamideacrylamide): Processing into Thin Films and Their Transitional Behavior," Polymer, 44, 7979-7988 (2003)

Simarmata, B. P., T. Listyani, R. A. Sukartono, and Simarmata; "Acid Mine Drainage Identification at Binuang Area, South Kalimantan, and Its Alternative Treatment," Jurnal Teknologi, 4, 113-119 (2011)

Zhu, C., J. Yun, Q. Wang, and G. Yang: “Adsorption of Ion Pairs onto Graphene Flakes and Impacts of Counterions during the Adsorption Processes," Applied Surface Science, 435, 329-337 (2018) 